\title{
Canine Leishmaniasis: Diagnosis by Cytology and Electron Microscopy.
}

\author{
Méndez, Adriana ${ }^{1 *}$, Ramos Godínez Pilar², Vázquez, Daniela ${ }^{1}$. \\ ${ }^{1}$ Universidad Nacional Autónoma de México, Facultad de Medicina Veterinaria y Zootecnia, \\ Departamento de Patología, CDMX, México. \\ ${ }^{2}$ Instituto Nacional de Cancerología, Patología, CDMX, México. \\ *Corresponding author:mvzadrimb@gmail.com.
}

Leishmaniasis is a disease transmitted by vectors and caused by the protozoan Leishamnia spp, whose main reservoirs are the human, dog, cat and other carnivores. The genus Leishmania is made up of trypanosomatid protozoa of the order kinetoplastide and include more than 20 different species. It is endemic in 90 countries with the exception of Australia and Antarctica, generally its distribution occurs in geographical areas with tropical or subtropical weather, which have optimal conditions for the survival and proliferation of the vector. The mosquitoes or sandflies of the genus Lutzomi in the new world and Phlebotomus in the old world, are the vectors responsible for the transmission. Leishmania exhibits two forms during its life cycle: a flagellated promastigote $(15-20 \mu \mathrm{m})$ found in the digestive system of the vector and an unflagellated amastigote $(3-5 \mu \mathrm{m})$ in the cytoplasm of host cells (macrophages and monocytes). Cutaneous leishmaniasis mainly associated with infections by L. donovani, L. tropica, L. infantum, complex L. mexicana spp. and L. baziliensis spp.

A medical consult was presented to the HVE - UNAM, a not sterilized female dog of 3.6 years of age, Spanish Water Dog breed, whose clinical history referred that she was obtained from a breeding place in Malaga Spain, since he was 3 months old. In the history, nasal secretion, ocular secretion, crusty lesions on the tip of both ears and epistaxis with 3 months of evolution were reported. At EFG, generalized lymphadenomegaly was found, nodule in the maxillary region of the right side of $1.4 \mathrm{~cm} \mathrm{x} 1.6 \mathrm{~cm}$ firm, subcutaneous and non-painful. Thin needle puncture of popliteal and left pre-scapular lymph nodes was performed. In the cytological exam an erythrocyte background witth abundant lymphocytes in several stages of maturation, moderate amount of histiocytes, plasma cells and limited amount of neutrophils was observed. In the cytoplasm of the histiocytes and extracellularly, parasitic structures compatible with amastigotes of Leishmania spp. A sample of popliteal lymph node for transmission electron microscopy was taken with the determination of evidencing the parasite and being able to make a conclusive diagnosis, since within the microscopic differential diagnoses to be considered were Trypanozoma cruzi, Histoplasma capsulatum and Toxoplasma gondii.

Cytology alone is a sensitive test for the diagnosis of lesihmaniasis as it allows to see the parasite and its morphology, within the macrophage cytoplasm. Also, the electron microscopy test allows you to fully evaluate the ultrastructure of the parasite (parasitic vacuole, nucleus, amastigote and kinetoplast).

Leishmaniasis is an endemic disease in Spain so it is very certain that the patient was infected when they arrived in the country, since the central area of Mexico is free of Leishmania spp, but not in states like Yucatán, Oaxaca and Chiapas.

So, the cytological diagnosis proves to be fast and conclusive in cases with clinical symptoms of leishmaniasis. The use of a complementary technique, such as electron microscopy, are essential for a conclusive diagnosis. 

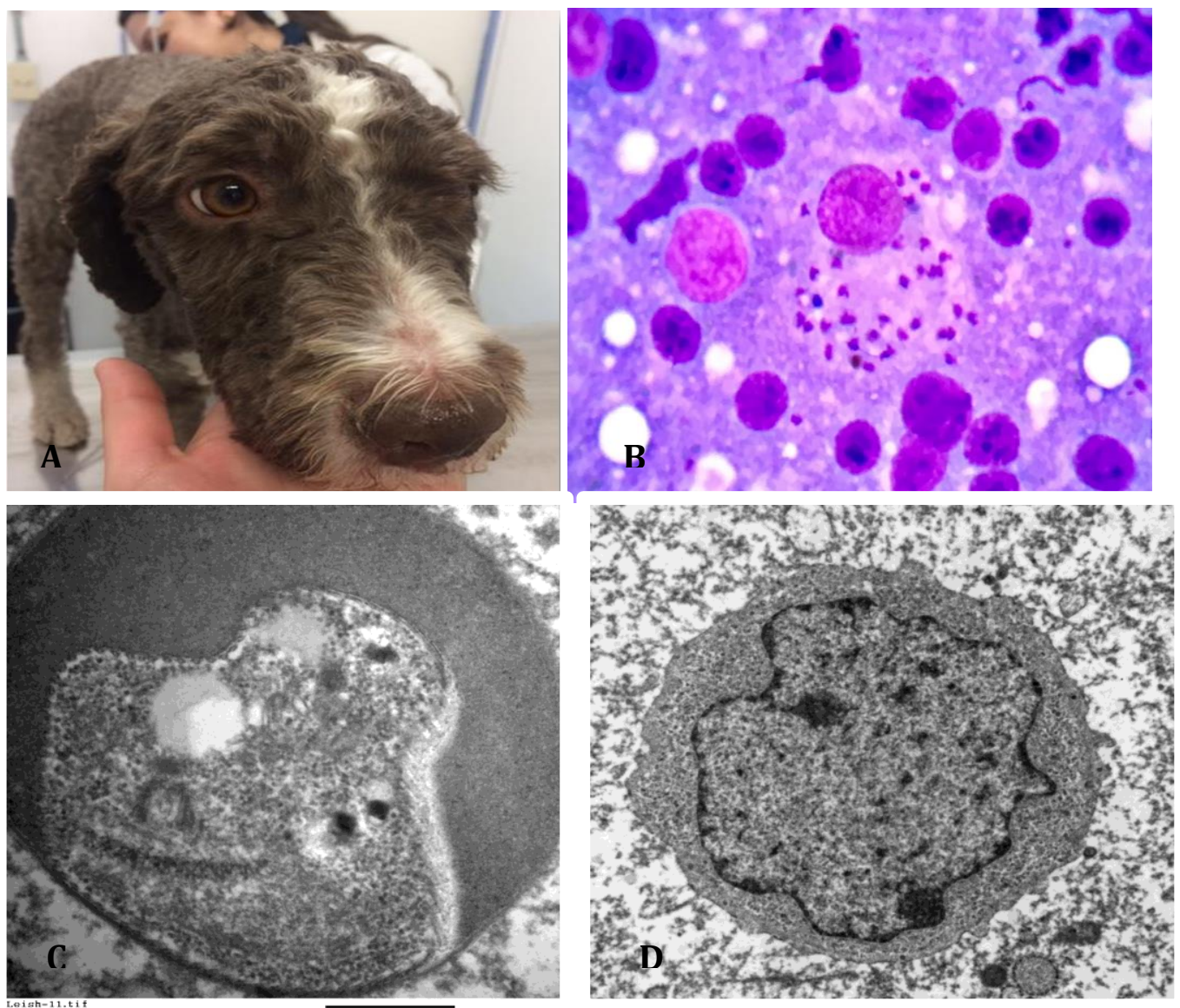

Figure 1. A. Photograph of the patient, shows erythematous lesions in the nasal plane. B. Photomicrograph of lymph node aspirate. Observe in the center a macrophage with numerous amastigotes. Adjacent to this, a lymphoblast and mature lymphocytes can be seen. Diff-Quick staining. C. Transmission electron photomicrograph of a amastigote, observe the kinetoplast (arrow). General contrast technique with uranyl acetate and lead citrate. $500 \mathrm{~nm}$ bar D. Transmission electronic photography. Observe a mature lymphocyte. Contrast technique with uranyl acetate and lead citrate. $500 \mathrm{~nm}$ bar.

\section{Reference.}

[1] Akhoundi M, et al. Leishmania infections: Molecular targets and diagnosis. Mol Aspects Med. (2017) p.12.

[2]Da Silva JGB, Guardao BJE, De Francesco DE. Kidney involvement in leishmaniasis-a review. Braz J Infect Dis. Volume18 (2014) p. 434-440.

[3] Kima EP. Leishmania molecules that mediate intracellular pathogenesis. Microbes infect. Volume 16 (2014) p.721-726.

[4] Mylonakis EM, et al. Cytologic patterns of lymphadenopathy in dogs infected with Leishmania infantum.Vet Clin Path. Volume 34 (2005) p. 243-247

[5] Pace D. Leishmaniasis. J Infect. Volume 69 (2014) p. 10-18.

[6] Valli T, Kiupel M, Bienzle D. 2016. Hematopoietic System. in: Jubb, Kennedy and Palmer's Pathology of domestic animals. (Elsiever, St. Louis, Missouri) p. 310-313.

[7] Zaidi A, Singh PK, Ali V. Leishmania and its quest for iron: An update and overview. Mol Biochem Parasitol. Volume 211 (2017), p. 15-25. 